\title{
Образование радиационных дефектов в слабо легированных слоях n- и $p$-SiC при торможении протонов
}

\author{
(C) В.В. Козловский ${ }^{1}$, А.Э. Васильев ${ }^{1}$, П.А. Карасев ${ }^{1}$, А.А. Лебедев ${ }^{2}$ \\ ${ }^{1}$ Санкт-Петербургский политехнический университет Петра Великого, \\ 195251 Санкт-Петербург, Россия \\ ${ }^{2}$ Физико-технический институт им. А.Ф. Иоффе Российской академии наук, \\ 194021 Санкт-Петербург, Россия \\ E-mail: kozlovski@physics.spbstu.ru
}

(Получена 9 октября 2017 г. Принята к печати 18 октября 2017 г.)

Путем математического моделирования каскада смещения в $\mathrm{SiC}$ рассмотрены особенности генерации пар Френкеля при рассеянии протонов с энергией 8 и 15МэВ. Рассчитаны гистограммы распределения энергий, полученных не только первично выбитыми атомами, но и атомами отдачи, генерируемыми в каскадах смещений. При анализе гистограмм рассмотрены две области энергий. В первой области „малых“ энергий преобладает процесс спонтанной рекомбинации генетически родственных пар Френкеля. Атомы отдачи второй области обладают большей энергией, позволяющей покинуть зону спонтанной рекомбинации и диссоциировать на изолированные компоненты. Проведено экспериментальное исследование процесса компенсации слабо легированных образцов $n$ - и $p-4 H-\mathrm{SiC}$, выращенных методом газофазовой эпитаксией, при облучении протонами с энергией 8 и 15 МэВ. Измерены скорости удаления носителей заряда. Проведено сравнение расчетных и экспериментальных данных и получены оценки размеров зоны спонтанной рекомбинации.

DOI: 10.21883/FTP.2018.03.45616.8741

\section{1. Введение}

Эффективность процесса вторичного радиационного дефектообразования в полупроводниках определяется прежде всего тем, насколько полно происходит диссоциация первично созданных радиационных дефектов пар Френкеля (ПФ): вакансия - межузельный атом. На устойчивость генетически родственной ПФ сильно влияет зарядовое состояние ее компонент [1-3]. Область неустойчивости в кристалле можно представить в виде сферы с радиусом, который называется радиусом рекомбинации $\left(r_{\text {rec }}\right)$ ПФ [4]. Таким образом, процесс диссоциации ПФ определяется в основном двумя факторами: распределением ПФ по расстоянию между компонентами и наличием электронов и дырок, обусловливающих перезарядку компонент. Атомы отдачи (AO) (recoils atoms), а следовательно, и ПФ могут образовываться либо при взаимодействии с протонами (так называемые первично выбитые атомы (ПВА) primary knockon (РКО) atoms), либо в каскадных процессах при взаимодействии энергетичных ПВА с атомами решетки (так называемые вторично выбитые атомы (BВА) secondary knock-on (SKO) atoms). Ранее в работах $[5,6]$ для случая облучения карбида кремния протонами мегаэлектронвольтных энергий было изучено распределение по энергии (и по расстоянию между компонентами ПФ) только первичных атомов отдачи. Такие расчеты нельзя назвать полными, так как большинство ПФ создается при протонном облучении именно в каскадах смещений. В настоящей работе решена более сложная задача анализ распределений по энергии всех атомов отдачи (total recoil atoms).

\section{2. Методика эксперимента}

Для численного моделирования торможения протонов в $\mathrm{SiC}$ использовался пакет программ SRIM [7], основанный на теоретических моделях, описанных в [8]. В ходе численного моделирования кроме гистограмм распределения по энергии атомов отдачи определялось количество вакансий в подрешетках кремния и углерода, а также полное число вакансий в расчете на падающий на пленку протон. Для пороговой энергии смещения атомов $\left(E_{d}\right)$ были взяты сравнительно новые в литературе значения 24 и 18 эВ для кремния и углерода соответственно $[9,10]$. Толщина пленки в расчетах принималась равной 50 мкм, согласно используемым в эксперименте образцам.

Протоны с энергией 8 и 15 МэВ использовались также для экспериментального наблюдения дефектов. Облучение проводилось на циклотроне МГЦ-20 при комнатной температуре. Частота импульсов 100 Гц, длительность импульса 2.5 мс. Средняя плотность тока пучка протонов составляла $10 \mathrm{HA} \cdot \mathrm{cm}^{-2}$. Дефекты вводились равномерно по объему пленки, поскольку ее толщина была значительно меньше длины пробега протонов. После облучения образцов определялась скорость удаления носителей заряда из зоны проводимости $\left(\eta_{e}\right)$ или из валентной зоны $\left(\eta_{p}\right)$ :

$$
\eta=\left(N_{0}-N\right) / D
$$

где $N_{0}$ и $N-$ концентрации носителей заряда в эпитаксиальном слое до и после облучения, $D-$ доза облучения. Изменения проводимости пленок определялись по вольт-емкостным характеристикам при температуре 
$T=300 \mathrm{~K}$ (на частоте 10 кГц). Образцы карбида кремния $4 H-\mathrm{SiC}$ представляли собой эпитаксиальные пленки толщиной 50 мкм, выращенные методом CVD в Германии (Leibniz-Institute for Crystal Growth, Berlin). Концентрация нескомпенсированной донорной (акцепторной) примеси в исходных пленках составляла $\sim 1 \cdot 10^{15} \mathrm{~cm}^{-3}$.

\section{3. Результаты и их обсуждение}

На рис. 1 представлен пример экспериментально полученных результатов: зависимость концентрации носителей заряда в $n$ - и $p-\mathrm{SiC}$ при облучении протонами с энергией 15 МэВ. Видно, что до значительных уровней компенсации полупроводников $(90 \%)$ как для $p-4 H-\mathrm{SiC}$, так и для $n-4 H-\mathrm{SiC}$ наблюдается линейная дозная зависимость. Линейность дозной зависимости предполагает, что уменьшение концентрации носителей заряда при облучении возникает за счет образования глубоких центров в объеме полупроводника, на которые уходят электроны (дырки) из зоны проводимости (валентной зоны) соответственно $[5,6]$. Причем в состав радиационных дефектов, образующих глубокие центры, могут входить компоненты пар Френкеля (вакансии и межузельные атомы) только собственных атомов полупроводника. Сравнительные данные по проявлению дефектов в компенсации проводимости $p-4 H-\mathrm{SiC}$ и $n-4 H-\mathrm{SiC}$ для использованных энергий протонов представлены в табл. 1. Отметим, что все скорости удаления носителей заряда, представленные в табл. 1, получены из линейных экспериментальных дозных зависимостей. Активность в удалении носителей заряда весьма показательна для характеристики радиационных дефектов.

Данные табл. 1 позволяют сделать следующие выводы. - Протоны с энергией $8 \mathrm{MэB} \mathrm{примерно} \mathrm{в} 2$ раза эффективнее протонов с энергией $15 \mathrm{MэB} \mathrm{в} \mathrm{отношении}$ компенсации проводимости как $n$-, так и $p$-SiC.

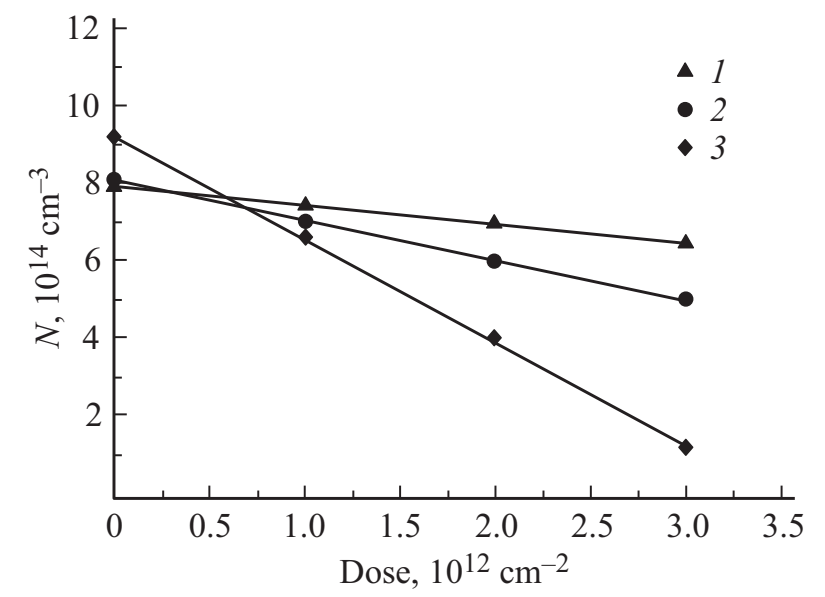

Рис. 1. Зависимость концентрации носителей заряда в $n-4 H-\mathrm{SiC}(1,2)$ и $p-4 H-\mathrm{SiC}(3)$ от дозы облучения протонами с энергией $15(1,3)$ и $8 \mathrm{MэB}(2)$.
Таблица 1. Скорость удаления носителей заряда в $n$-SiC $\left(\eta_{e}\right)$ и $p-\mathrm{SiC}\left(\eta_{p}\right)$ при облучении протонами различных энергий

\begin{tabular}{c|r|l}
\hline Облучение & $\eta_{e}, \mathrm{~cm}^{-1}$ & $\eta_{p}, \mathrm{~cm}^{-1}$ \\
\hline Протоны $8 \mathrm{MэB}$ & 100 & $480-500$ \\
Протоны $15 \mathrm{MэB}$ & 55 & $240-260$
\end{tabular}

Таблица 2. Количество смещений, созданных в пленке $\mathrm{SiC}$ толщиной 50 мкм в первичных и вторичных столкновениях, в расчете на один протон

\begin{tabular}{c|c|c|c|c|c}
\hline $\begin{array}{c}E_{p}, \\
\text { МэВ }\end{array}$ & $\begin{array}{c}\text { Подре- } \\
\text { шетка }\end{array}$ & $\begin{array}{c}\text { Первично } \\
\text { выбитые } \\
\text { атомы (РКО) }\end{array}$ & $\begin{array}{c}\text { Вторично } \\
\text { выбитые } \\
\text { атомы (SKО) }\end{array}$ & $\begin{array}{c}\text { Сумма } \\
\text { вакансий в } \\
\text { подрешетке }\end{array}$ & $\begin{array}{c}\eta_{\mathrm{FP}}, \\
\mathrm{CM}^{-1}\end{array}$ \\
\hline \multirow{2}{*}{8} & $\mathrm{Si}$ & 0.59 & 1.24 & 1.83 & 366 \\
& $\mathrm{C}$ & 0.34 & 1.54 & 1.88 & 376 \\
& $\mathrm{Si}$ & 0.30 & 0.66 & 0.96 & 192 \\
& $\mathrm{C}$ & 0.17 & 0.82 & 0.99 & 198
\end{tabular}

- Скорость удаления дырок $\eta_{p}$ примерно в 5 раз выше, чем скорость удаления электронов $\eta_{e}$ для обеих энергий используемых протонов.

Поскольку компенсация проводимости полупроводника определяется глубокими центрами, созданными компонентами ПФ, скорость изменения проводимости непосредственно является характеристикой степени диссоциации генерированных ПФ. Далее мы проведем численное моделирование кинематики каскадов смещений и сопоставим с экспериментальными результатами для торможения протонов. Сравнение с аналитическими выражениями мы будем проводить, используя классическую формулу резерфордовского рассеяния для получения атомом отдачи с атомным номером А и зарядовым числом $\mathrm{Z}$ энергии, большей $E_{d}$ :

$$
\begin{aligned}
\sigma_{d} & =\left(1 / 4 \pi \varepsilon_{0}\right)^{2} \pi\left[Z^{2} e^{4} m_{p} / M E_{p} E_{d}\right] \\
& =2.6 \cdot 10^{11} Z^{2} /\left[4 A E_{p}(э \mathrm{~B}) E_{d}(э \mathrm{~B})\right] \text { (барн). }
\end{aligned}
$$

В табл. 2 приведены результаты численного моделирования: количество вакансий в подрешетках кремния и углерода, а также полное число вакансий в расчете на падающий на пленку протон. В третьей графе табл. 2 приведен результат столкновений протонов с атомами $\mathrm{Si}$ и С. Следующая графа отражает количество вакансий, созданных в подрешетках $\mathrm{Si}$ и С атомами отдачи. При этом, например, атомы $\mathrm{Si}$ могут выбиваться из узлов атомами отдачи любой природы (в равной степени и $\mathrm{Si}$, и C). В последней колонке табл. 2 представлена скорость генерации пар Френкеля $\eta_{\mathrm{FP}}$ (одним из компонентов которой является вакансия) в единицах $\mathrm{cm}^{-1}$. Укажем, что анализ проводился при статистике 100 тысяч упавших на пленку протонов. 
Данные табл. 2 позволяют сделать следующие выводы. - С уменьшением энергии протонов примерно в 2 раза (с 15 до $8 \mathrm{MэB)} \mathrm{в} \mathrm{соответствии} \mathrm{с} \mathrm{законом}$ резерфордовского рассеяния (2) в такое же число раз должно увеличиться и сечение столкновений $-\sigma$ и как следствие количество вакансий, создаваемых непосредственно протоном. Согласно данным, приведенным в третьей графе, эта закономерность хорошо прослеживается для атомов обеих подрешеток.

- Другая характеристика резерфордовского закона зависимость $\sigma \propto Z^{2} / A E_{d}$ - очень хорошо просматривается в количестве рассеяний протона на атомах $\mathrm{Si}$ и С (см. графу 3 табл. 2). Согласно этой зависимости, количество вакансий, созданных непосредственно протонами в подрешетке кремния, в 1.75 раза больше, чем в подрешетке углерода.

- Однако в каскадном процессе значительно больше вакансий создается уже в подрешетке углерода. Так что суммарное количество вакансий, созданных протонами и атомами отдачи, в обеих подрешетках практически одинаково (графа 5 табл. 2).

- При протонном облучении доля вакансий, созданных непосредственно протонами, составляет $17 \%$ для подрешетки углерода. Остальные $83 \%$ создаются атомами отдачи в каскадных процессах. Для подрешетки кремния эти цифры составляют 31 и $69 \%$ соответственно. Поэтому процессы компенсации проводимости $\mathrm{SiC}$ будут определяться глубокими центрами, созданными не ПВА, а атомами отдачи, образованными в каскадах смещений.

На рис. 2 представлена зависимость количества смещений (PKO и SKO), создаваемых в первичных и вторичных процессах, от энергии протонов.

Теперь перейдем к сравнению расчетных и экспериментальных данных. Начнем с компенсации проводимости $n-\mathrm{SiC}$ при протонном облучении. При сравнении необходимо учесть следующее обстоятельство. В работах [9-11] с помощью низкоэнергетического (до 200 кэВ) электронного облучения было показано, что основные радиационные дефекты (РД), обусловливающие компенсацию проводимости слабо-легированного $n$ - $\mathrm{SiC}$ (CVD) (так называемые $Z 1 / Z 2-$ и $E H 6 / 7$-дефекты), связаны с компонентами ПФ только в одной из подрешеток $\mathrm{SiC}$, а именно - в углеродной. В работе [12] аналогичные предположения были сделаны для объяснения результатов влияния протонного облучения на компенсацию проводимости $n$-SiC. Поэтому мы будем сравнивать расчетную скорость генерации пар Френкеля только в подрешетке углерода с экспериментально измеренной скоростью удаления носителей заряда. Поскольку в пленке толщиной 50 мкм один протон образует в среднем 0.99 вакансий углерода, скорость введения пар Френкеля $\eta_{\mathrm{FP}}$ (в обычно используемых единицах см${ }^{-1}$ ), составит $198 \mathrm{~cm}^{-1}$ (см. табл. 2). Напомним, что экспериментально полученное значение $\eta_{e}$ составляет $55 \mathrm{~cm}^{-1}$. Таким образом, при облучении протонами с энергией

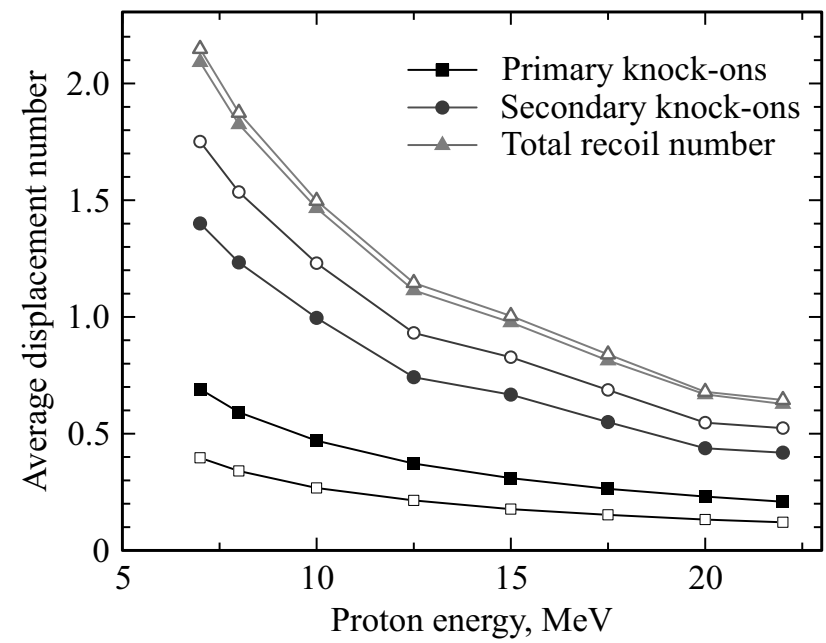

Рис. 2. Зависимость количества смещений, создаваемых в пленке $\mathrm{SiC}$ в расчете на один протон, от энергии протонов: сплошные символы - смещения в подрешетке кремния; пустые символы - смещения в подрешетке углерода. Расчет по программе SRIM [7] для случая облучения образцов $\mathrm{SiC}$ толщиной 50 мкм.

$15 \mathrm{MэB}$ в $n$-SiC диссоциирует примерно четверть генерирующихся вакансий углерода. Сравнивая значения $\eta_{e}=100 \mathrm{~cm}^{-1}$ и $\eta_{\mathrm{FP}}=376 \mathrm{~cm}^{-1}$, полученные для энергии протонов 8 МэВ (табл. 1 и 2), мы убедились, что и в этом случае диссоциирует примерно четверть созданных вакансий углерода.

Для анализа процесса диссоциации ПФ необходимо рассчитать энергию, передаваемую в актах столкновений. Причем не только в столкновениях с протонами, как сделано в работах $[5,6]$, но и, что более важно, в каскадных процессах, поскольку большинство ПФ образуется именно в каскадах смещений. Напомним, что анализ проводился при статистике 100 тысяч упавших на пленку протонов. Толщина пленки принималась равной 50 мкм, согласно используемым в эксперименте образцам.

На рис. 3 приведена плотность распределения по энергии атомов отдачи углерода для случая облучения $\mathrm{SiC}$ протонами с энергией 8 и $15 \mathrm{MэB.}$

Зафиксированы столкновения, приведшие к возникновению вакансий, т.е. передачи малых (сравнительно с порогом смещения атома) энергий не учитываются. Суммарное число столкновений составило 99000 , что, на наш взгляд, достаточно для выяснения общей конфигурации спектра. Нижняя граница спектра рис. 3 совпадает с пороговой энергией 18 эВ дефектообразования; верхняя граница ограничена значением 200 эВ, обеспечивающим попадание в интервал спектра $93 \%$ атомов отдачи. Поясним, что наблюдавшиеся в ходе моделирования акты $(\sim 7 \%)$ с передачей энергии большими порциями (до десятков кэВ) в поле рис. 3 не попадают и обсуждаются далее. Огибающая линия энергетического спектра ПВА (плотности распределения ПВА), изме- 


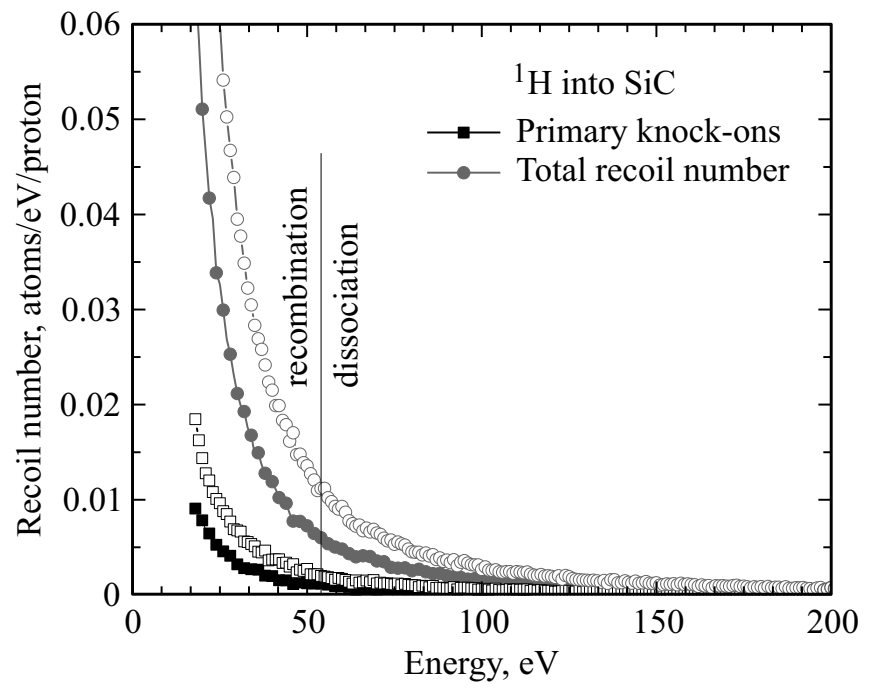

Рис. 3. Плотность распределения по энергии атомов отдачи углерода для случая облучения $\mathrm{SiC}$ протонами с энергией 8 (пустые символы) и 15 (сплошные символы) МэВ. Вертикальной линией указана энергия $(57$ э), делящая спектр на высокоэнергетическую область (зона диссоциации ПФ) и низкоэнергетическую область (зона рекомбинации ПФ). Расчет по программе SRIM [7] для случая облучения образцов $\mathrm{SiC}$ толщиной 50 мкм.

няется согласно закону резерфордовского рассеяния, обратно пропорционально квадрату переданной энергии. Из сравнения гистограмм ПВА и АО видно, что распределение АО спадает значительно круче, чем ПВА.

Учитывая, что в эксперименте диссоциирует четверть образующихся вакансий углерода, попробуем определить граничную энергию атома отдачи углерода, которая делит спектр в отношении 3:1. Согласно расчетам, эта граничная энергия имеет значение 57 эВ. Несколько удивительно, что эта энергия, согласно нашим расчетам, является граничной и для спектров атомов отдачи, полученных при других энергиях налетающих протонов в диапазоне 5-25 МэВ. Граничная энергия обозначена на рис. 3 вертикальной линией, делящей спектр на высокоэнергетическую область (зона диссоциации ПФ) и низкоэнергетическую область (зона рекомбинации ПФ). Атомы отдачи углерода, получившие энергию 57 эВ и выше, будут покидать зону спонтанной рекомбинации, обеспечивая формирование стабильных РД, приводящих к компенсации проводимости $n$-SiC. Используя результаты расчетов по программе SRIM, легко оценить пробег ионов углерода с энергией 57 эB в $\mathrm{SiC}$. Этой энергии соответствует пробег, равный $\sim 6 \AA$, который можно приписать радиусу рекомбинации $\left(r_{\text {rec }}\right)$. Поскольку расстояние между ближайшими атомами углерода в $4 H-\mathrm{SiC}$ составляет $a_{0} \sim 1.85 \AA$ [13-15], радиус рекомбинации составляет примерно величину, равную трем $a_{0}$.

Интересно сравнить полученное значение $r_{\text {rec }}$ ПФ $\mathrm{SiC}$ с литературными данными по кремнию. В работах [16-18] указывается, что в кремнии (n-Si) при электронном облучении диссоциируют только 5\% первичных ПФ; поэтому $r_{\text {rec }}$ оценивается величиной порядка $20 \AA$. Для протонного облучения кремния литературных данных по $r_{\text {rec }}$ нет; поэтому мы провели расчеты по программе SRIM, аналогичные вышеописанным расчетам для SiC. На рис. 4 представлена плотность распределения по энергии атомов отдачи для случая облучения кремния протонами с энергией 15 МэВ. В связи с известной дискуссией о величине $E_{d}$ для кремния нами выполнены расчеты для двух значений $E_{d}$, наиболее часто встречающихся в литературе: 13 и 25 эВ $[4,6,8,16]$. Расчеты показали, что скорость введения ПФ составляет $250 \mathrm{~cm}^{-1}$ (для $E_{d}=25$ эВ) и $500 \mathrm{~cm}^{-1}$ (для $E_{d}=13$ эВ). Экспериментально измеренная скорость удаления электронов $\eta_{e}$ составляет, по данным $[16], 120 \mathrm{~cm}^{-1}$. Таким образом, степень диссоциации ПФ составляет $50 \%$ для больших значений $E_{d}$ и $25 \%$ для малых значений $E_{d}$. Оценим граничную энергию атома отдачи, при которой в правой части энергетических спектров AO, представленных на рис. 3, будет находиться половина и четверть АО соответственно. Оказалось, что для обоих спектров эта энергия приблизительно одинакова и составляет 45 эВ. Пробег ионов кремния с такой энергией в кремнии составляет $8 \AA$, который можно приписать радиусу рекомбинации $\left(r_{\text {rec }}\right)$ ПФ. Таким образом, при идентичных условиях протонного облучения $r_{\text {rec }}$ ПФ в кремнии несколько выше $(8 \AA)$, чем $r_{\text {rec }}$ ПФ в подрешетке углерода $\operatorname{SiC}(6 \AA)$.

Далее оценим рекомбинацию ПФ в подрешетке углерода, имеющую место при электронном облучении образцов $\mathrm{SiC}$, аналогичных используемым в настоящей работе. Сравнивая полученные в работах $[19,20]$ значения $\eta_{e}=0.1 \mathrm{~cm}^{-1}$ и $\eta_{\mathrm{FP}}=1 \mathrm{~cm}^{-1}$ для энергии электронов 0.9 МэВ, можно сказать, что при электронном

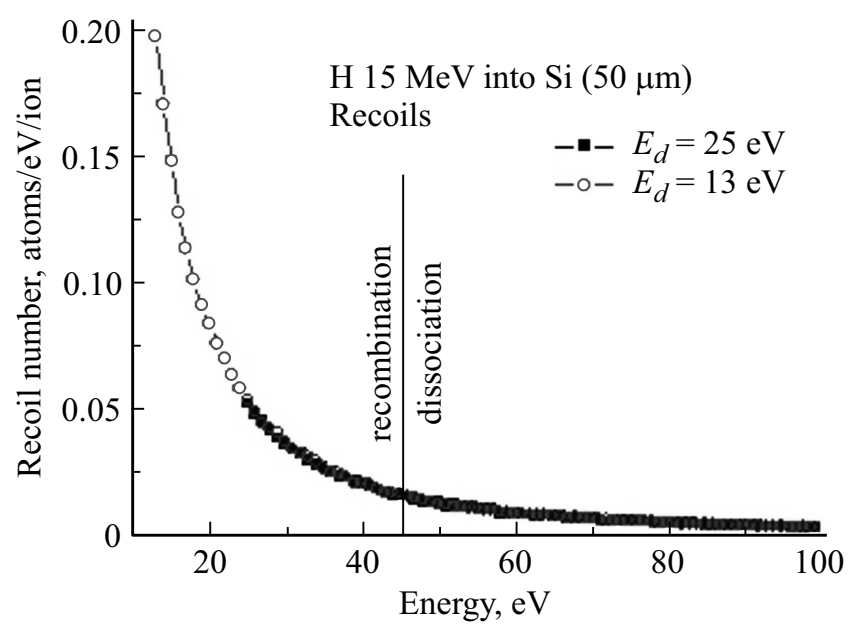

Рис. 4. Плотность распределения по энергии атомов отдачи кремния для случая облучения кремния протонами с энергией $15 \mathrm{MэB}$, полученная из расчетов по программе SRIM [7] для значений $E_{d}$, равных 13 и 25 эВ. Вертикальной линией указана энергия (45 эВ), делящая спектр на высокоэнергетическую область (зона диссоциации ПФ) и низкоэнергетическую область (зона рекомбинации ПФ). 
облучении диссоциирует примерно $10 \%$ созданных ПФ. Энергетический спектр АО при электронном облучении совпадает со спектром ПВА, так как каскадные процессы практически отсутствуют. Поскольку спектр ПВА подчиняется аналитическим резерфордовским формулам $[21,22]$, по ним легко определить граничную энергию АО, делящую спектр в отношении $9: 1$. Она оказалась равной 120 эВ. Соответственно радиус рекомбинации увеличивается до $\sim 9 \AA$, т.е. до пяти $a_{0}$. Мы предполагаем, что увеличение радиуса рекомбинации ПФ как в $\mathrm{SiC}$, так и в $\mathrm{Si}$ при электронном облучении связано с изменением зарядового состояния компонент ПФ. Так, для кремния известно, что нейтральные компоненты ПФ имеют радиус рекомбинации $\sim 2 a_{0}$, а компоненты, заряженные разноименными зарядами, $\sim 7 a_{0}[6,16,17]$.

Оценим темп генерации электронно-дырочных пар (ЭДП) в карбиде кремния при облучении протонами на импульсном циклотроне МГЦ-20 и электронами на импульсном ускорителе РТЭ-1В $[19,20]$. Для энергии протонов $15 \mathrm{MэB}$ неупругие потери энергии, рассчитанные по программе SRIM, составляют 8.6 кэВ/мкм. Энергия образования ЭДП в $\mathrm{SiC}$ составляет $8.4-8.6$ эВ $[23,24]$. Тогда один протон генерирует в начале трека электронно-дырочные пары со скоростью $1000 \mathrm{мкм}^{-1}$ или $1 \times 10^{7} \mathrm{~cm}^{-1}$. При использованной в настоящей работе плотности потока протонов в импульсе $\sim 2.4 \cdot 10^{11} \mathrm{~cm}^{-2} \cdot \mathrm{c}^{-1}$ полный темп генерации ЭДП может составлять $\sim 2.4 \cdot 10^{18} \mathrm{~cm}^{-3} \cdot \mathrm{c}^{-1}$. Для протонов с энергией $8 \mathrm{MэB} \mathrm{неупругие} \mathrm{поте-}$ ри составляют $\sim 14$ кэВ/мкм, а темп генерации ЭДП $3.9 \cdot 10^{18} \mathrm{~cm}^{-3} \cdot \mathrm{c}^{-1}$. Облучение электронами в работах $[19,20]$ выполнялось на импульсном ускорителе РТЭ-1В. Частота импульсов 450 Гц, длительность импульса 370 мкс, скважность 6. Пучок с током 1 мА и диаметром сечения 0.9 см сканирует по площади $2 \times 40$ см. Средняя плотность тока пучка при облучении электронами считается $12.5 \mathrm{M \kappa A} \cdot \mathrm{cm}^{-2}$, однако в импульсе плотность тока значительно выше и составляет $6 \mathrm{MA} \cdot \mathrm{cm}^{-2}$. Для энергии электронов $0.9 \mathrm{MэВ} \mathrm{неупругие}$ потери энергии составляют 0.75 кэВ/мкм. Тогда один электрон генерирует в начале трека электрон-дырочные пары со скоростью $87 \mathrm{мкм}^{-1}$ или $8.7 \cdot 10^{5} \mathrm{~cm}^{-1}$. Поскольку плотность потока электронов в импульсе составляет $3.6 \cdot 10^{16} \mathrm{~cm}^{-2} \cdot \mathrm{c}^{-1}$, темп генерации ЭДП при облучении электронами достигает величины $3 \cdot 10^{22} \mathrm{~cm}^{-3} \cdot \mathrm{c}^{-1}$. Таким образом, при облучении электронами темп генерации ЭДП на 4 порядка выше, чем при протонном облучении. Этот фактор может приводить к перезарядке компонент ПФ, образующихся при электронном облучении, и как следствие увеличению в 1.5 раза радиуса рекомбинации.

Таким образом, для объяснения процесса компенсации проводимости карбида кремния $n$-типа проводимости при протонном и электронном облучении вполне достаточно простейшей модели, связывающей компенсацию с образованием пар Френкеля только в подрешетке углерода. Достаточно, чтобы от 10 до $25 \%$ из общего числа образовавшихся ПФ диссоциировали из зоны спонтанной рекомбинации.

К сожалению, такая простая модель не подходит для объяснения экспериментальных результатов по карбиду кремния $p$-типа проводимости. В $p$ - $\mathrm{SiC}$ экспериментально наблюдаемая величина $\eta_{p} \sim(480-500) \mathrm{cm}^{-1}$, напри-

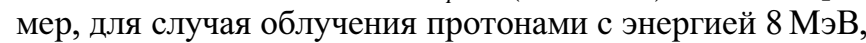
значительно превосходит расчетное значение скорости генерации пар Френкеля как в подрешетке углерода $\left(376 \mathrm{~cm}^{-1}\right)$, так и подрешетке кремния $\left(366 \mathrm{~cm}^{-1}\right)$. Поэтому за компенсацию проводимости отвечают первичные дефекты в обеих подрешетках. Сильное различие в поведении (степени диссоциации) ПФ в материалах $p$ и $n$-типа проводимости может быть связано не только с различными подрешетками, но и с зарядовым состоянием компонент ПФ, образующихся в материалах $p$ и $n$-SiC.

\section{4. Заключение}

В ходе проведенных исследований было установлено, что компенсация карбида кремния $n$ - и $p$-типа проводимости при облучении протонами носит линейный характер от дозы облучения вплоть до полной компенсации. Полученные результаты указывают на то, что основным механизмом компенсации является переход свободных носителей на глубокие центры, связанные с образующимися при обучении собственными первичными радиационными дефектами. Проведенные в работе численные расчеты показали, что результаты экспериментальной компенсации проводимости $n$ - $\mathrm{SiC}$, облученного протонами с энергией 8 и $15 \mathrm{MэB}$, хорошо объясняются генерацией вакансий только в подрешетке углерода. При этом виде облучения всего $25 \%$ пар Френкеля избегают рекомбинации и разделяются на отдельные компоненты, входящие в состав известных $Z 1 / Z 2-$ и $E H 6 / 7$-дефектов, которые приводят и к изменению электрофизических свойств материала (в частности, электропроводимости), измеряемых в эксперименте. Радиус рекомбинации ПФ в подрешетке углерода, по нашим оценкам, составляет $\sim 6 \AA$, а энергия АО углерода, необходимая для получения пробега $6 \AA$, составляет 57 эВ.

Настоящая работа выполнена при поддержке гранта РНФ № 16-12-10106.

\section{Список литературы}

[1] A.A. Lebedev. Radiation Effects in Silicon Carbide (Material Research Forum LLC, Millersville, PA 17551, USA, v. 6 (2017). ISSN 2471-8890; ISBN 978-1-945291-11-1).

[2] G. Lucas, L. Pizzagalli. J. Phys.: Codens. Matter, 19, 086208 (2007).

[3] V.V. Emtsev, V.Yu. Davydov, I.N. Goncharuk, V.V. Kozlovski, E.V. Kalinina, D.S. Poloskin, A.V. Sakharov, N.M. Shmidt, A.N. Smirnov, A.S. Usikov. Mater. Sci. Forum, 258-263, 1143 (1997). 
[4] В.В. Емцев, Т.В. Машовец, В.В. Михнович. ФТП, 27, 708 (1993).

[5] В.В. Козловский, А.Э. Васильев, А.А. Лебедев. Поверхность, 3, 22 (2015).

[6] А.М. Иванов, В.В. Козловский, Н.Б. Строкан, А.А. Лебедев. ФТП, 45, 145 (2011).

[7] http://www.srim.org

[8] J.F. Ziegler, J.P. Biersack, U. Littmark. The Stopping and Range of Ions in Matter (Pergamon, N.Y., 1985).

[9] J.W. Steeds, F. Carosella, G.A. Evans, M.M. Ismail, L.R. Danks, W. Voegeli. Mater. Sci. Forum, 353-356, 381 (2001).

[10] J.W. Steeds, G.A. Evans, S. Furkert, M.M. Ismail, L.R. Danks, W. Voegeli, F. Carosella. Diamond Relat. Mater., 11, 1923 (2002).

[11] H. Kaneko, T. Kimoto. Appl. Phys. Lett., 98, 262106 (2011).

[12] A. Castaldini, A. Cavallini, L. Rigutti. Semicond. Sci. Technol., 21, 724 (2006).

[13] M.E. Levinshtein, S.L. Rumyantsev, M.S. Shur (eds). Properties of Advanced Semiconductor Materials: GaN, AlN, InN, $B N$, SiC, SiGe (John Wiley \& Sons, Inc., 2001).

[14] N. Iwamoto, B.G. Svensson. Point Defects in Silicon Carbide. Chap. 10. In: Defects in Semiconductors, ed. by L. Romano, V. Privitera, Ch. Jagadish. (Ser. Semiconductors and Semimetals) (Elsevier, 2015) v. 96, p. 369.

[15] A. Holmes-Siedle, L. Adams. Handbook of radiation effects (Oxford, Oxford University, 1993).

[16] V.V. Emtsev, A.M. Ivanov, V.V. Kozlovski, A.A. Lebedev, G.A. Oganesyan, N.B. Strokan, G. Wagner. ФТП, 46, 473 (2012).

[17] A.M. Strel'chuk, V.V. Kozlovski, N.S. Savkina, M.G. Rastegaeva, A.N. Andreev. Mater. Sci. Engin. B, 61-62, 441 (1999).

[18] V.A.J. Van Lint. Mechanisms of Radiation Effects in Electronic Materials (John Wiley \& Sons,1980).

[19] V.V. Kozlovski, A.A. Lebedev, E.V. Bogdanova. J. Appl. Phys., 117, 155702 (2015).

[20] Н.Б. Строкан, А.М. Иванов, Н.С. Савкина, В.В. Козловский, А.А. Лебедев, М. Syvajarvi, R. Yakimova. ФТП, 38, 841 (2004).

[21] В.В. Козловский, В.В. Емцев, К.В. Емцев, Н.Б. Строкан, А.М. Иванов, В.Н. Ломасов, Г.А. Оганесян, А.А. Лебедев. ФТП, 42, 243 (2008).

[22] А.А. Лебедев, А.И. Вейнгер, Д.В. Давыдов, Н.С. Савкина, А.М. Стрельчук, В.В. Козловский. ФТП, 34, 897 (2000).

[23] M. Rogalla, K. Runge, A. Soldner-Rembold. Nucl. Phys. B., 78, 516 (1999).

[24] A. Castaldini, A. Cavallini, F. Nava, P.G. Fuochi, P. Vanni. Mater. Sci. Forum., 433-436, 439 (2003).

\section{Formation of radiation defects in the moderately doped layers when braking protons}

\author{
V.V. Kozlovski ${ }^{1}$, A.E. Vasil'ev ${ }^{1}$, P.A. Karaseov ${ }^{1}$, \\ A.A. Lebedev ${ }^{2}$
}
${ }^{1}$ Peter the Great St. Petersburg Polytechnic University, 195251 St. Petersburg, Russia
${ }^{2}$ loffe Institute,
194021 St. Petersburg, Russia

Abstract Peculiarities of Frenkel pair generation by 8 and $15 \mathrm{MeV}$ protons in $\mathrm{SiC}$ are investigated experimentally and by modeling of displacements in individual collision cascades. The process of conductivity compensation in moderately doped $n$ and $p$ - $4 H$-SiC samples grown by vapour-phase epitaxy by 8 and $15 \mathrm{MeV}$ proton irradiation was studied experimentally. The charge carrier removal rates are measured. The energy histograms are drawn for the primary knock-on atom distributions and for total amount of recoils generated in collision cascades. Histograms obtained were analyzed as consisting of two parts. The first part (,small“ energies) corresponds to predomination of the process of spontaneous recombination of genetically related Frenkel pairs. The recoil atoms fall to the second part has more energy, which allow them to leave the area of spontaneous recombination and thus Frenkel pairs dissociate into isolated components, able to form various defect complexes in the target. Spontaneous recombination area dimensions are estimated on the base of comparison of calculated and experimental data.

Редактор Г.А. Оганесян 